\title{
Avaliação da qualidade perceptiva dos sinais de voz transmitidos sob diferentes condições de canal sem fio
}

\author{
Perceptual quality evaluation of speech signals transmitted under different wireless channel \\ conditions
}

Evaluación de la calidad de percepción de las señales de voz transmitidas en diferentes condiciones de canal inalámbrico

\section{Resumo}

Nos serviços de comunicação, a qualidade e integridade do sinal de voz no receptor é um fator relevante que as operadoras de telefonia devem considerar. Neste artigo, estudamos o impacto da degradação que ocorre no canal de transmissão no sinal de voz transmitido. Para tanto, são utilizados parâmetros-chave, como a pontuação média de opiniões (MOS) relacionada ao sinal de voz e a taxa de erro de bit (BER), a fim de avaliar qualitativa e quantitativamente o sinal de fala em cada cenário. É usado um cenário de simulação de modulação, que considera os canais de desvanecimento AWGN, Rician e Rayleigh, e duas modulações: BPSK e QAM. Esses canais têm diferentes parâmetros de configuração. Os resultados experimentais mostram que existem certos valores de índice de qualidade MOS para cada tipo de canal com uma configuração específica, mostrando que para cada cenário de transmissão há a necessidade de adaptações na combinação modulação-canal-ganho para obter melhor desempenho.

Palavras-chave: BPSK; 4QAM; MOS; BER; Canal AWGN; Canal de Rician; Canal de Rayleigh.

\begin{abstract}
In communication services, the quality and integrity of the voice signal at the receiver is a relevant factor that telephone operators must consider. In this article, we study the impact of degradation that occurs in the transmission channel on the transmitted voice signal. Therefore, key parameters are used, such as the average opinion score (MOS) related to the voice signal and the bit error rate (BER), in order to qualitatively and quantitatively evaluate the speech
\end{abstract}


signal in each scenario. A modulation simulation scenario is used, which considers the AWGN, Rician and Rayleigh fading channels, and two modulations: BPSK and QAM. These channels have different configuration parameters. Experimental results show that there are certain MOS quality index values for each type of channel with a specific configuration, showing that for each transmission scenario there is a need for adaptations in the modulation-channelgain combination to obtain better performance.

Keywords: BPSK; 4QAM; MOS; BER; AWGN Channel; Rician Channel; Rayleigh Channel.

\section{Resumen}

En los servicios de comunicación, la calidad e integridad de la señal de voz en el receptor es un factor relevante que los operadores telefónicos deben considerar. En este artículo estudiamos el impacto de la degradación que se produce en el canal de transmisión sobre la señal de voz transmitida. Por tanto, se utilizan parámetros clave, como la puntuación media de opinión (MOS) relacionada con la señal de voz y la tasa de error de bit (BER), para evaluar cualitativa y cuantitativamente la señal de voz en cada escenario. Se utiliza un escenario de simulación de modulación, que considera los canales de desvanecimiento AWGN, Rician y Rayleigh, y dos modulaciones: BPSK y QAM. Estos canales tienen diferentes parámetros de configuración. Los resultados experimentales muestran que existen ciertos valores de índice de calidad MOS para cada tipo de canal con una configuración específica, mostrando que para cada escenario de transmisión hay una necesidad de adaptaciones en la combinación modulación-canalganancia para obtener un mejor desempeño.

Palabras clave: BPSK; 4QAM; MOS; BER; Canal AWGN; Canal Rician; Canal Rayleigh.

\section{Introdução}

Devido ao avanço da tecnologia e ao processo de globalização, cada vez se percebe a necessidade de transmissão de mensagens de forma eficiente, prezando pela sua velocidade e ausência de perdas de dados. O desenvolvimento tecnológico, em especial os sistemas de telecomunicações digitais vêm passando por transformações nas comunicações de voz, dados e vídeo. Verifica-se a necessidade de avaliar o desempenho das redes de telecomunicações, tais como qualidade do sinal transmitido, explorando potenciais falhas que degradam o sinal em seu percurso. Para essa avaliação de desempenho das redes de telecomunicações, podemos citar parâmetros chave de análise (key parameters) tais como BER e MOS, comumente citadas na literatura como suporte estatístico na análise de perdas (error) de dados no sinal transmitido. Em telecomunicações, MIMO é um método utilizado em sistemas de comunicação sem fio para multiplicar a capacidade de transmissão utilizando múltiplas antenas de transmissão e recepção, explorando a propagação multi caminho (multipath).

O BER (Bit Error Rate) ou Taxa de Erros de Bits é definida como a porcentagem de bits que apresentam erros em relação ao número total de bits recebidos em uma transmissão. BER é geralmente expresso como 10 elevado a uma potência negativa. Por exemplo, se uma transmissão tem um BER de 10 elevado a menos 4, isso significa que de 10.000 bits transmitidos, 1 teve um erro. Um valor alto de BER indica uma linha com ruído, o que pode causar baixo desempenho da rede. Devido a necessidade de mitigar os efeitos indesejados do desvanecimento (fading) ou variações na potência do sinal recebido em transmissão de dados, a qualidade na comunicação tendeu-se a aumentar com o passar dos anos, mantendo-se a baixa complexidade dos dispositivos transmissores e receptores e ao mesmo tempo evitando aumento da demanda de potência transmitida (Vieira, 2005).

A diversidade espacial divide-se em diversidade de recepção, onde se utilizam duas ou mais antenas no receptor canal MISO (Multiple Input Single Output), e diversidade de transmissão, onde se utilizam duas ou mais antenas no transmissor canal SIMO (Single Input Multiple Output). Considera-se que a diversidade de recepção tenha sido amplamente explorada, o que ao contraste com a diversidade de transmissão, que tem sido objeto de constante exploração atualmente com uso de códigos espaço-temporais. Visando a possibilidade de alcance de maiores taxas de transmissão de dados, o sistema MIMO de Múltiplas Entradas Múltiplas Saídas no setor de comunicações veio caracterizado como um conjunto de antenas nos transmissores e receptores que se baseiam na diversidade espacial de transmissão de dados (Ghani, Dixit \& Wang, 2000).

Para empregar sistemas MIMO, implementa-se os códigos espaço-temporais e executa-se o processamento de conjuntos de antenas na recepção (Vieira, 2005). Com o aumento das pesquisas na área, os sistemas MIMO agora apresentam 
capacidade de incremento linear em função do número de antenas transmissoras e receptoras. Isso faz com que sistemas MIMO para comunicações banda larga seja uma tendência futura, onde a modelagem de um canal pode ser utilizado para estimar parâmetros do sinal recebido pelo receptor, identificando a quantização da perda de dados no enlace de transmissão.

Quando tratamos de tecnologias de eficiência energética e sistemas autônomos, lembramos que Ong e Lambotharan (2006) propuseram uma taxa adaptativa em alocação de energia para serviços integrados de voz e dados em sistemas MIMO. Segundo os autores, a potência disponível no transmissor MIMO é limitada, onde o serviço de voz recebe maior prioridade, pois a potência apropriada é alocada para satisfazer a BER. Nesse sentido desenvolve-se um sistema de adaptação baseada na potência média e taxa ideal para eficiência espectral máxima em função da BER. Foi ideal o desenvolvimento de um esquema de taxa fixa e controle de energia em sistema SISO, em que a transmissão de dados é empregada em taxa adaptativa (baseado em M-QAM).

Pelo fato da existência de distúrbios tais como interrupção, Ramprashad, Pepin e Kozat (2006) descrevem uma estrutura que pode ser usada para orientar na avaliação mais realista da qualidade de mídia na presença de eventos não desejados em sistemas MIMO sem fio, bem como a otimização de seu transporte, e sugere que as estratégias de transmissão MIMO que agregam diversidade (vinculadas à fonte) podem apresentar benefícios para a qualidade da mídia quando comparadas a MIMO que se concentram apenas na capacidade. A capacidade desses sistemas na transmissão de sinais por subcanais independentes já é uma realidade. Podemos citar que Ong e Lambotharan (2007) propõem um serviço integrado de voz e dados para um sistema MIMO que atribui um subcanal para transmissão de voz e os demais canais para transmissão de dados, alocando voz e dados em diversas configurações, o que pôde ser visto com uma quebra nos paradigmas vigentes da época. Com base na distribuição de autovalores de matrizes aleatórias de Wishart, pode-se obter expressões para controle de taxa de potência no intuito de analisar o desempenho sob indicadores de QoS (Quality of Service).

Uma técnica adaptativa empregando constelações de modulação de amplitude em quadratura M-hierárquica (MQAM) com codificação de bloco de espaço-tempo (STBC) foi explorada por Li, Zhang e Lu (2008) como solução de camada de enlace para voz simultânea e em diferentes classes de problemas de transmissão de dados em MIMO. Apresenta-se o BER de transmissão de voz e dados através de canais de desvanecimento MIMO Nakagami-M correlacionados, e como resultado obtém-se maior eficiência na modulação QAM. As interferências (portadoras) são indesejáveis em sistemas de comunicação, sendo necessário soluções menos complexas. Já Kanthimathi e Kavitha (2011) abordam o LTE (Long Term Evolution) que emprega MIMO-OFDM em algoritmo de sincronização da frequência (portadora) para o sistema lidar com as problemáticas citadas anteriormente.

Com o aumento da necessidade por sistemas mais robustos, Lien e Chen (2011) adotam um modelo no qual implementam um esquema de controle de tráfego, compreendendo a programação de transmissão de pacote e o controle de admissão para LTE em sistemas WLAN. A avaliação de desempenho BER em comunicações reais sob diferentes canais de comunicação, tais como AWGN, Rayleigh e Rician são abordados por Awon, Islam, Rahman e Islam (2012), onde se constrói um modelo da camada física WIMAX para aplicação de diferentes taxas de codificação e modulação digital em dados sintéticos para os canais. Isso se torna útil para investigar os efeitos dos canais em sistemas semelhantes baseado na IEEE 802.16 (OFDM), onde medidas de desempenho podem ser baseadas em BER x Eb/No.

A implementação de sistemas OFDM com modulação BPSK e QAM sobre modelos de canais AWGN, Rician e Rayleigh cresceram nos últimos anos como uma poderosa ferramenta de análise de qualidade do sinal, na busca constante por obtenção de alta eficiência espectral e melhores resistências à ambientes de desvanecimentos (Borra \& Chaparala, 2013). Sistemas MIMO-OFDM possuem alta taxa de transmissão e robustez contra desvanecimento de múltiplos (Vidhya \& Kumar, 2013). Vale salientar que a implementação de modelos AWGN, Rician e Rayleigh em diferentes métodos de estimativa de canal como LS (Least Square), LS-Mod (Least Square-Modified) e MMSE (Minimum Mean Square Error), podem ser ótimas 
ferramentas para análise de desempenho baseado em parâmetro BER.

Algoritmos baseados em normas internacionais como ITU (International Telecommunication Union) agregam maior tecnologia e robustez em projetos de transmissão, assim como Parida, Das e Paul (2013), que apresentam um algoritmo para escalonamento VoIP semi-persistente usando modo CLR-1 MIMO (Closed Loop Rank-1 MIMO), no qual o desempenho é avaliado de acordo com as metodologias de teste especificadas pela ITU, bem como a previsão do uso de PRB (Physical Resource Bloc) e a capacidade VoIP para CLR-1.

O uso de CR (Cognitive Radio) na melhoria da eficiência da utilização do espectro em modulação BPSK sob canais AWGN, Rician, Rayleigh e Nakagami-M por meio de parâmetro BER já é conhecido (Mohammed, 2014). Verifica-se vantagens com uso do AWGN em comparativos, mas não documentados detalhadamente. Já Nomura, Mori, Naito e Kobayashi (2014) trabalharam na redução do tempo do canal especial e no fornecimento de melhores indicadores de QoS para comunicação de voz em WLAN (Wireless Local Area Network).

Pelas análises dos autores citados, verifica-se que a escolha da melhor modulação e canal se dá em virtude do cenário escolhido. Isso pode ser observado nos estudos de Ahlem, Dadi e Rhaimi (2015) que utilizaram modulação em comunicação sem fio com intuito de verificar o desempenho de canais por meio de BER sob canais AWGN, Rician e Rayleigh, no qual obtiveram melhor desempenho com canal Rician. Observamos que sistemas MIMO também podem ser utilizados em metodologias ECC (Envelope Correlation Coefficient). Os Coeficientes de Correlação de Envelope nos dizem o quão independentes são os padrões de radiação entre duas antenas, medindo o nível de correlação uma com a outra. Podem ser implementadas sob a medida da SAR (Specific Absorption Rate). A Taxa de Absorção Específica é comumente usada no intuito de medir a energia que é absorvida por unidade de massa por um corpo humano quando exposto a um campo eletromagnético de RF (Radio Frequency) (Agrawal \& Srivastava, 2017).

Técnicas de modulação QAM variável foram explorados (Mohammed \& Bilal, 2017) sob o parâmetro BER como medida de desempenho em sistema CR. Com modelos de canais AWGN, Rician e Rayleigh, os autores Mohammed, et al., (2017) usaram modulação variável (ordem 4-16-64) em range de $30 \mathrm{~dB}$, onde obtiveram melhores resultados com os dois primeiros modelos. A análise de desempenho em QoE em simulações de PLR (Packet Loss Rate) com uso do parâmetro MOS foram analisados por Affonso, Rosa e Rodríguez (2018). A observação da Taxa de Perda de Pacotes sob esse parâmetro tem emergido como alternativas aos modelos convencionais de análises de voz. O uso dessas tecnologias aliada a determinadas técnicas de modulações está estreitamente ligada às últimas tendências de transmissão de dados como 5G (Kabir, Rahman \& Ullah, 2018), em que seu sistema de transmissão de sinal de frequência de voz é mais seguro e rápido (compatível com downlink MIMO NOMA sem fio em sistema de comunicação) no intuito foi desenvolver um sistema eficaz na recuperação do sinal de áudio (QAM).

A análise de desempenho com o MOS tratada em estudos por Rodríguez, Pívaro, Rosa, Mittag e Möller (2018), no qual avaliam a degradação da qualidade de voz em interface wireless sob a tecnologia MIMO sob ITU-T P.862 e P.563. Para essa métrica é desejável a maximização do score MOS, e a definição de limiar mínimo para avaliação da qualidade do áudio. O uso de padronizações internacionais em telecomunicações também é explorado por Rodríguez, Silva, Silva e Junior (2018), que abordam a degradação de sinal em redes sem fio por meio do impacto de diferentes mudanças na DF (Doppler Frequency) nas comunicações de fala. O uso de modelos de canal de desvanecimento em diferentes perfis de atraso de múltiplo caminho no uso da recomendação ITU-T (com parâmetros P.563 e P.862) podem ser utilizados para avaliar o desempenho da qualidade da fala.

O desempenho da qualidade de voz em QoS sob parâmetros da ITU-T como modelo de sistema MIMO baseado em sistema wireless trabalham com como sinal/ruído, deslocamento Doppler, configurações MIMO e diferentes tipos de modulações (Rodríguez, Rosa, Almeida, Mittag \& Möller, 2019). O algoritmo ITU-T Rec. P.862.2 foi usado na avaliação das 
amostras de falas juntamente com código IW-M que incorpora a consideração da qualidade por sistema MIMO de diferentes arranjos, por meio da inclusão de valores no score $R$ Global, assim acredita-se que sistemas MIMO podem usar múltiplas antenas no transmissor e receptor no intuito de minimizar o BER, maximizando a qualidade do sinal.

\section{Metodologia}

\subsection{Definindo parâmetros}

O objetivo do método proposto nesse trabalho é o de analisar e comparar graficamente as diferenças entre o comportamento MOS e BER para uma amostra de áudio em 3 tipos de canais de desvanecimento: AWGN, Rician e Rayleigh. De modo geral primeiro define-se os parâmetros dos testes laboratoriais. Foi definido 50 experimentos por cada ciclo de processamento. Na primeira etapa escolheu-se o modelo BPSK de ordem 2. Na segunda etapa escolheu-se o modelo QAM de ordem 4. Para ambas etapas de teste definiu-se o range de ruído AWGN entre $-10 \mathrm{~dB}$ até $20 \mathrm{~dB}$. A quantidade de níveis de quantização 216 foi fixado. Cada um desse modelos de configuração foi aplicado aos canais 0:AWGN, 1:Rician, 2:Rayleigh.

\subsection{Backup e quantização linear do sinal}

Em nosso sistema, após cada interação é criado um backup contendo as informações do horário de criação, no intuito de separar as amostras geradas. O algoritmo faz a varredura no diretório local de execução e verifica se há o arquivo executável P563 ou P862. Identificando-o, automaticamente cria-se uma pasta chamada Resultados para nosso sistema alocar os dados de saída coletados. Em seguida, o sistema verifica a relação sinal ruído (SNR), carregando o arquivo de áudio original de teste inserido no modelo. Assim, ocorre a quantização linear do sinal e logo a conversão para binário e arranjo de bits em sequência. Aplica-se o código de Hamming, criando o objeto de sistema modulador de acordo com o definido inicialmente nos parâmetros (BPSK ou 4QAM).

\subsection{Modulação/Demodulação}

Modulando-se os dados, eles estão prontos para serem aplicados ao canal de desvanecimento desejado, gerando o gráfico do sinal transmitido e recebido. Após isso ocorre a demodulação do sinal, no qual coleta-se os erros estatísticos do sistema. Ocorre a sequência para agrupação e conversão de binário para inteiro e reverte-se a quantização, gerando-se a imagem do áudio. Salva-se o sinal do áudio recebido para novas análises laboratoriais aplicando o teste MOS de qualidade para QoE, e plota-se os MOS/BER médios obtidos. Finalizando podemos salvar o áudio na pasta desejada com os atributos de nomeação definidos para organização mais adequada das informações. O código fonte do projeto pode ser obtido pelo link: https://github.com/davidaugustoribeirobrazil/speech-signals.

\section{Resultados e Discussão}

O modelo desenvolvido foi construído usando uma amostra de arquivo de áudio, que teve por objetivo servir como modelo a ser transmitido com a finalidade de analisar os efeitos de sua degradação pelas técnicas de modulação. O presente trabalho mostrará os melhores resultados em função dos canais abordados. Caso o leitor deseje ver desempenho de outros canais pode acessar o link do código anteriormente citado para matlab e Simulink. Para técnica de modulação BPSK foram utilizadas as seguintes configurações: Modulação BPSK e canal AWGN sob aspecto de análise MOS x Eb/No conforme a Figura 1 e gerado graficamente a simulação do sinal transmitido. 
Figura 1: Qualidade do sinal de áudio (MOS) para esquema de modulação BPSK em Canal de Desvanecimento AWGN, e Gráfico do sinal de áudio transmitido e recebido.
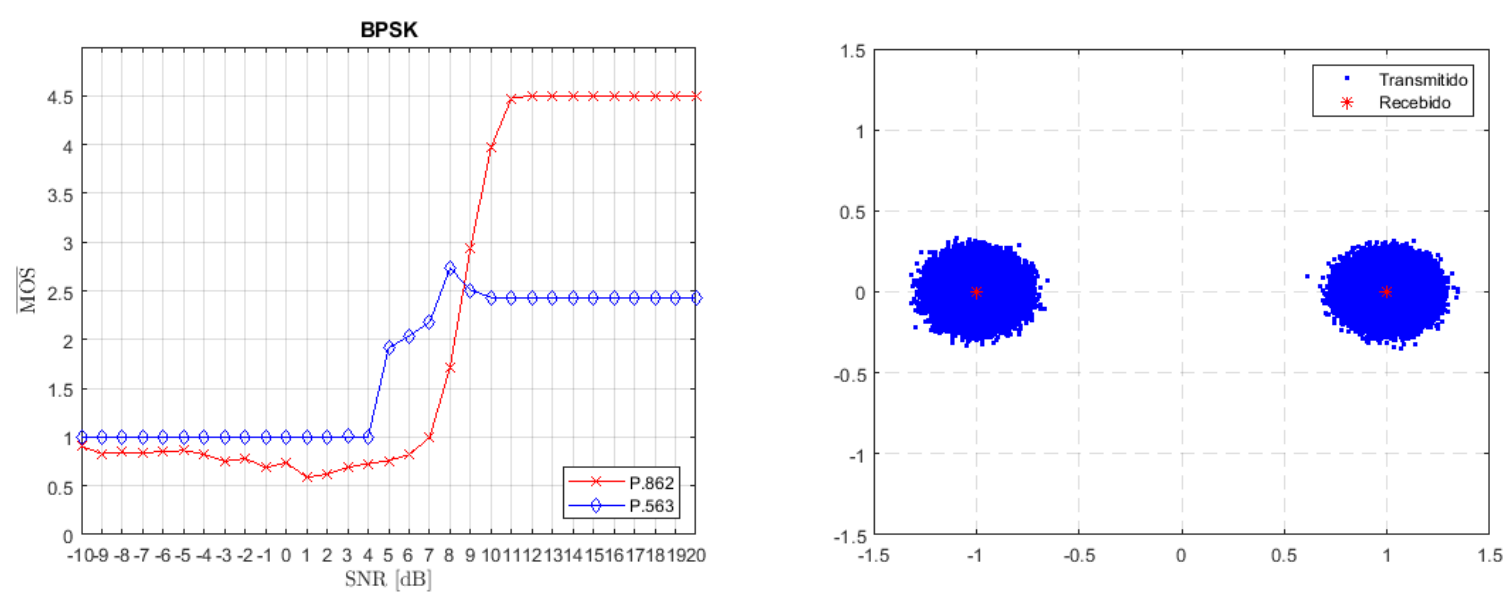

Fonte: Autores (2021).

O canal que apresentou maior otimização para resposta MOS nos dados transmitidos foi o AWGN Fading Channel (Figura 1), no qual a partir de $11 \mathrm{~dB}$ apresenta o nível MOS de 4.5. Para técnica de modulação 4QAM foram utilizadas as seguintes configurações: Modulação BPSK e canal 4QAM sob aspecto de análise MOS x Eb/No conforme a Figura 2 e gerado graficamente a simulação do sinal transmitido.

Figura 2: Qualidade do sinal de áudio (MOS) para esquema de modulação 4QAM em Canal de Desvanecimento AWGN, e Gráfico do sinal de áudio transmitido e recebido.
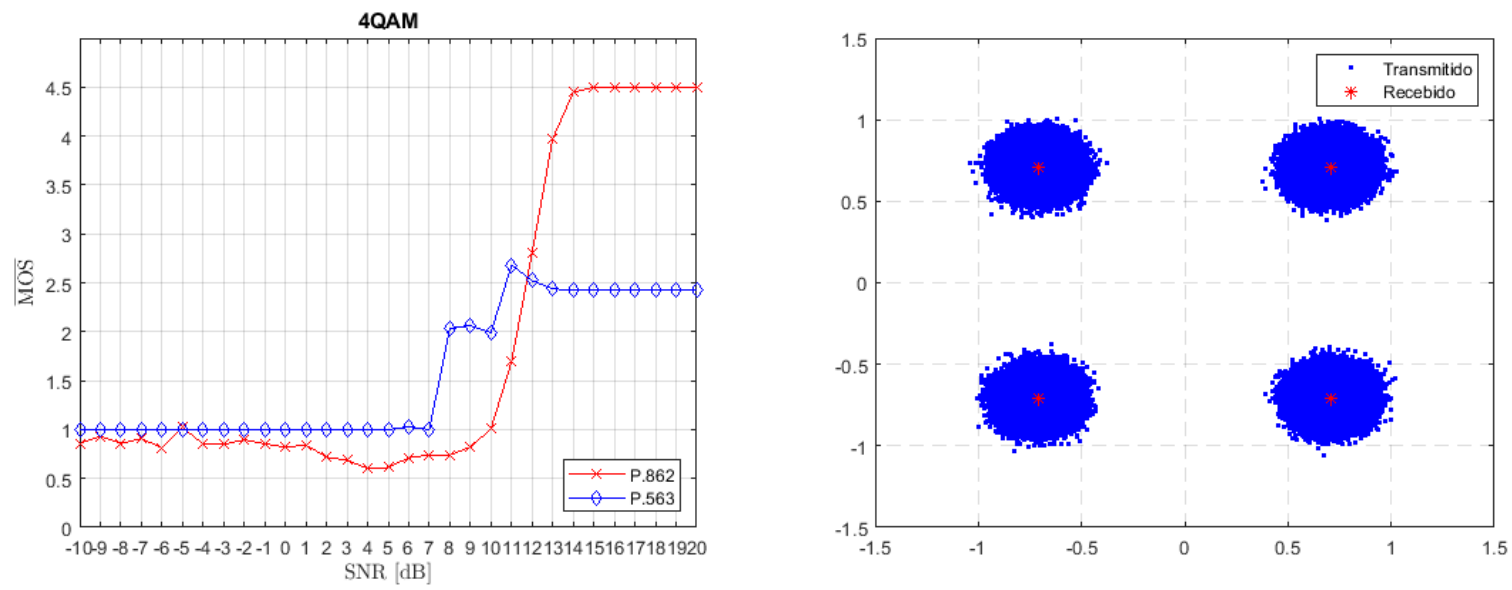

Fonte: Autores (2021).

De acordo com os gráficos, o canal que apresentou maior otimização para resposta MOS nos dados transmitidos foi novamente o AWGN Fading Channel, no qual a partir de $14 \mathrm{~dB}$ apresenta o nível MOS de 4.5. Foi desenvolvido em esquemáticos a Figura 3 com intuito de obter análises BER do sistema de áudio degradado anterior para sistemas de modulação BPSK e 4QAM respectivamente. 
Figura 3: Estrutura em Simulink matlab para Sistema BPSK/4QAM MIMO.
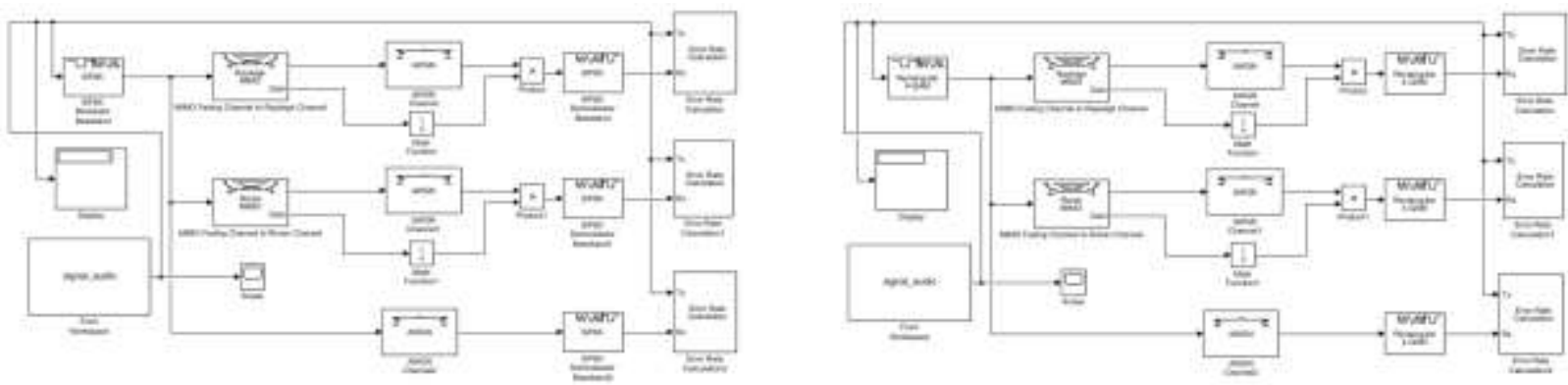

Fonte: Autores (2021).

Figura 4: BER em Canais AWGN, Rician e Rayleigh em modulação BPSK MIMO.

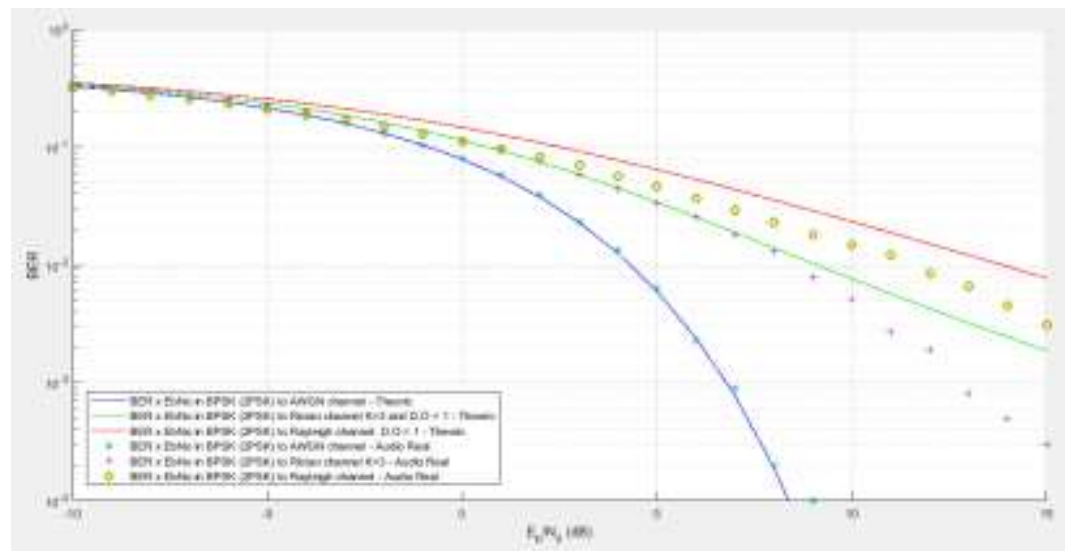

Fonte: Autores (2021).

Figura 5: BER em Canais AWGN, Rician e Rayleigh em modulação 4QAM MIMO.

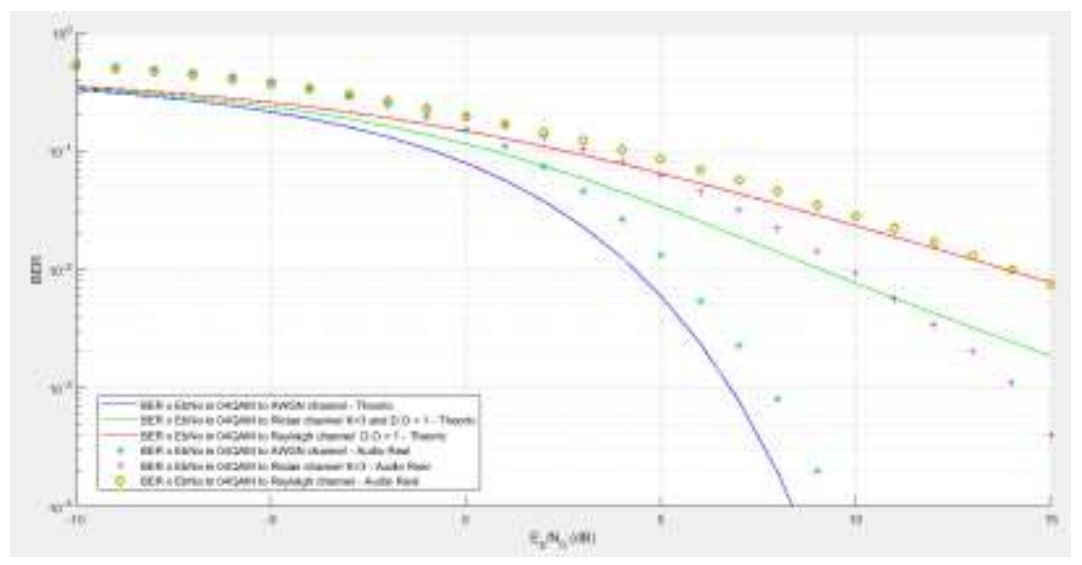

Fonte: Autores (2021).

O ruído introduzido foi calculado com base na energia em termos de Eb/No ou densidade espectral de energia para ruído de energia na unidade dB para o módulo de canal AWGN. O módulo de demodulação BPSK é executado. O sinal recebido é então colocado no MCTE (Módulo de Cálculo de Taxa de Erro), que calcula a taxa de erro para a transmissão do sinal. Esse bloco tem a tarefa de fazer comparativos entre o sinal (bruto) da quantização binária dos dados de áudio de entrada com os dados modulados adicionados com ruídos (gaussianos). O sistema compila o resultado da taxa de erro, em uma operação que divide o número total de erros pelo número total de bits. Portanto, a saída do bloco MCTE que recebe os dados processados, o transmite para a workspace para serem trabalhados pela toolbox bertool. Por meio dos esquemáticos 
desenvolvidos, foram realizados testes de comparativos entre sinais teóricos e reais, no qual percebe-se uma grande proximidade de comportamento entre eles (Figuras 4 e 5). Essa semelhança se dá pelo fato da alta robustez de ambas as técnicas de modulação empregadas, mesmo possuindo baixa ordem.

\section{Considerações Finais}

A problemática da perda de dados em transmissão de sinal de voz é algo bem frequente ainda em tecnologias atuais como em celulares e computadores. Há momentos em que o ruído pode sobrepor a voz do emissor, causando uma diminuição na compreensão da fala e assim causando a diminuição do índice QoS. Certas ocasiões podem haver falhas e perda completa do sinal, fazendo com que o emissor fique completamente "mudo" em relação ao receptor. Fatores ambientais locais influenciam na transmissão/recepção do sinal e ao mesmo tempo também o tipo de canal empregado na transmissão. Portanto, necessita-se estabelecer quais canais são mais indicados em modelos específicos de estrutura de rede. Por meio das simulações, pode-se afirmar com os resultados obtidos, que o parâmetro chave MOS/BER em sistemas de transmissão de sinal de voz são parâmetros importantes no que tange a análise da integridade dos dados transmitidos por meio dos canais de desvanecimento abordados neste trabalho. As técnicas de modulação BPSK/4QAM escolhidas se mostraram robustas pois apresentam similaridade no comportamento tanto do sistema simulado com entrada geradora de sinal randômico (gerada no bertool), como com o de entrada de áudio (modelo real), o que leva a crer que a arquitetura empregada é eficaz para analisar esse tipo de dados (no cenário específico). Observa-se por meio dos gráficos que o BER diminui em função do aumento da energia do $\mathrm{Eb} / \mathrm{No}(\mathrm{dB})$ pelo fato de que quanto maior a ordem da modulação, maior será o bit para uma transmissão. O canal de desvanecimento AWGN em modulação BPSK é o mais eficiente em termos de BER x Eb/No do que o 4QAM, em virtude do: aumento de complexidade do tipo de modulação, ordem da modulação, aumento proporcional da taxa de dados. A maior demanda de energia (Eb/No) do sistema 4QAM foi no intuito de manter o BER para um valor limitante definido. Sendo assim, obteve-se a análise qualitativa (MOS) e quantitativa (BER) do sistema no cenário simulado, o que levou o projeto a contribuir para os estudos das técnicas de transmissão e recepção de sinais de voz com sistemas MIMO que estão em forte ascensão. Ressalta-se, entretanto a limitação da técnica de modulação BPSK para transmissão em altas taxas de dados, necessitando-se assim de técnicas de modulação de ordem mais elevada para manutenção da estabilidade. Com o advento dos processamentos em GPU/TPU com alto poder na execução de cálculos, novos projetos são sugeridos a serem realizados futuramente, de modo que estes estejam voltados a avaliar aplicações em outros modelos de modulações (ordem superior) e com os canais de desvanecimentos abordados no trabalho, no intuito de avaliar seu grau de desempenho frente aos modelos no estado da arte disponíveis.

\section{Agradecimentos}

Os autores agradecem a CAPES (Coordenação de Aperfeiçoamento de Pessoal de Nível Superior) pelo suporte e fomento.

\section{Referências}

Affonso, E. T., Rosa, R. L., \& Rodríguez, D. Z. (2018). Speech quality assessment over lossy transmission channels using deep belief networks. IEEE Signal Processing Letters, 25, 70-74

Agrawal, T., \& Srivastava, S. (2017). Compact mimo antenna for multiband mobile applications. Journal of Microwaves, Optoelectronics and Electromagnetic Applications (JMOe), 16, 542-552

Ahlem, B. L., Dadi, M. B., \& Rhaimi, C. B. (2015). Evaluation of ber of digital modulation schemes for awgn and wireless fading channels. World Congress on Information Technology and Computer Applications (WCITCA), 1-5

Awon, N. T., Islam, M. A., Rahman, M. M., \& Islam, A. Z. M. T. (2012). Effect of AWGN \& fading (raleigh \& rician) channels on BER performance of a wimax communication system. CoRR 
Borra, S. K., \& Chaparala, S. K. (2013). Performance evaluation of ofdm system with rayleigh, rician and awgn channels. International Journal of Emerging Technology and Advanced Engineering, vol. 3, Mar.

Ghani, N., Dixit, S., \& Wang T.S. (2000). On ip-over-wdm integration. IEEE Communications Magazine, 38, 72-84

Kabir, M. H., Rahman, J., \& Ullah, S. E. (2018). Secured voice frequency signal transmission in 5g compatible multiuser downlink mimo noma wireless communication system. International Journal of Networks and Communications, 8, 97-105

Kanthimathi, M., \& Kavitha, C. (2011). Improved performance by ici cancellation in mimo-ofdm system. International Conference on Signal Processing, Communication, Computing and Networking Technologies, 111-115

Li, G., Zhang, Z., \& Lu, Z. (2008). Adaptive hierarchical modulation over correlated mimo fading channels. 9th International Conference on Signal Processing, 1920-1925

Lien, S. Y., \& Chen, K. C. (2011). Statistical traffic control for cognitive radio empowered lte-advanced with network mimo. IEEE Conference on Computer Communications Workshops (INFOCOM WKSHPS), 80-84

Mohammed, A. G. A. (2014). Performance evaluation of bpsk modulation-based spectrum sensing over wireless fading channels in cognitive radio. IOSR Journal of Electronics and Communication Engineering, 9, 24-28

Mohammed, A. I., \& Bilal, K. H. (2017). Impact of awgn, rayleigh and rician fadingchannels on ber performance of a cognitive radio network. International Journal of Scientific Engineering Research, 8, 1365-1368

Nomura, Y., Mori, K., Naito, K., \& Kobayashi, H. (2014). High efficient packet aggregation scheme for multi-rate and voip packet transmissions in next generation mu-mimo wlans. International Conference on Advanced Technologies for Communications (ATC 2014), 517-521

Ong, L. T., \& Lambotharan, S. (2006). Variable rate variable power mimo system for integrated voice and data services. 10th IEEE Singapore International Conference on Communication Systems, 1-5

Ong, L. T., \& Lambotharan, S. (2007). On the rate and power allocation for mimo based integrated voice and data transmission. IEEE $66^{\text {th }}$ Vehicular Technology Conference, 1762-1766

Parida, P., Das, S. S., \& Paul, S. (2013). Performance of voip in presence of mimo interference in ofdma (lte) networks. IEEE $77^{\text {th }}$ Vehicular Technology Conference (VTC Spring), 1-6

Ramprashad, S. A., Pepin, C., \& Kozat, U. C. (2006). Distortion tradeoffs of different layered speech and media transmission techniques over wireless mimo systems. Fortieth Asilomar Conference on Signals, Systems and Computers, 1308-1315

Rodríguez, D. Z., Pívaro, G. F., Rosa, R. L., Mittag, G., \& Möller, S. (2018). Quantifying the quality improvement of mimo transmission systems in voip communication. 26th International Conference on Software, Telecommunications and Computer Networks (SoftCOM), 1-5

Rodríguez, D. Z., Silva, M. J., Silva, F. J. M., \& Junior, L. C. B. (2018). Assessment of transmitted speech signal degradations in rician and rayleigh channel models. INFOCOMP Journal of Computer Science, 17, 23-31

Rodríguez, D. Z., Rosa, R. L., Almeida, F. L., Mittag, G., \& Möller, S. (2019). Speech quality assessment in wireless communications with mimo systems using a parametric model. IEEE Access, 7, 35719-35730

Vidhya, K., \& Kumar, K. S. (2013). Ber performance of awgn, Rayleigh and rician channel. International Journal of Advanced Research in Computer and Communication Engineering, 2, 2058-2067

Vieira, L. F. (2005). Análise do efeito da propagação em canais com múltiplas entradas e múltiplas saídas (mimo) com base no traçado de raios. Master's thesis, Pontifical Catholic University of Rio de Janeiro. 